\title{
O Programa de Educação pelo Trabalho para Saúde (PET-Saúde) como indutor de inovações pedagógicas: a experiência do curso de Odontologia da Universidade Estadual de Feira de Santana, Bahia
}

\author{
Graciela Soares Fonsêca*, Ana Áurea Alécio de Oliveira Rodrigues** \\ * Mestranda do Programa de Ciências Odontológicas - área de \\ concentração Odontologia Social da Faculdade de Odontologia da \\ Universidade de São Paulo (FOUSP) \\ ** Mestre em Saúde Coletiva/Professa Assistente da Universidade \\ Estadual de Feira de Santana (UEFS)
}

\section{RESUMO}

A evolução das práticas odontológicas e as novas concepções sobre a efetividade da atenção em saúde bucal exigem profissionais críticos, capazes de trabalhar em equipe e de levar em conta a realidade social. A proposta é conceber cirurgiões-dentistas com perfil generalista, com sólida formação técnico-científica, humanística e ética, orientada para a promoção de saúde. Nesse propósito, mudanças estão sendo instituídas no ensino superior, buscando a integração com os serviços de saúde e com a comunidade, para favorecer o processo de ensino-aprendizagem. Cita-se o Programa de Educação pelo Trabalho para a Saúde, o PET-Saúde, como forte indutor de inovação pedagógica. O presente trabalho relata a experiência da Universidade Estadual de Feira de Santana, Bahia, salientando as mudanças geradas no Curso de Odontologia, no intuito de oferecer subsídios para que o programa seja implementado e aperfeiçoado em outras localidades do país. Observa-se que foram alcançados resultados significativos com a incorporação do PET-Saúde, evidenciando o potencial transformador, o que coopera para sua consolidação e expansão.

\section{DESCRITORES}

Ensino superior. Serviços de saúde. Odontologia.

\begin{abstract}
$\Delta$ Odontologia praticada no serviço público, no Brasil, se caracterizou, durante um longo período, por serviços prestados de maneira assistemática e pela livre demanda de pacientes, ${ }^{1}$ marcado por procedimentos mutiladores, baseados na queixa-conduta e caracterizados como não resolutivos.

Como reflexo dessa forma de organizar a assistência, a formação em Odontologia, por anos, entendeu a saúde como resultado do processo biológico, fundada em princípios flexnerianos ${ }^{1}$ e distante do contexto social. Os conceitos que significaram grandes evoluções na compreensão do processo saúde-doença, entendendo a saúde como um bem socialmente determinado, tardaram a atingir o ensino de Odontologia. Por conseqüência, o modelo de formação visava uma atenção odontológica elitizada, desvinculada da noção de bem-estar e de qualidade de vida, dirigida a uma necessidade de saúde ressentida por todos, mas acessível apenas a elite financeira da população. Assim, relegou-se ao ensino da odontologia o desenvolvimento técnico, sendo por muito tempo denominado de arte dentária, onde se aproximam os termos arte e técnica. ${ }^{2}$

Aponta-se esse fator como um dos responsáveis pela precariedade das condições de saúde bucal da população brasileira, ${ }^{3}$ em que a mutilação dentária
\end{abstract}

\footnotetext{
${ }^{1}$ Modelo baseado em um paradigma fundamentalmente biológico e mecanicista para interpretação dos fenômenos vitais, com culto à doença e não à saúde, e devoção à tecnologia, sob a presunção de que seria o centro da atividade científica e de assistência à saúde.
} 
marca fortemente adultos e idosos, retratando as necessidades acumuladas e não priorizadas ${ }^{4}$.

O início do século XXI foi marcado pela redefinição da Política Nacional de Saúde Bucal, ${ }^{4}$ pressupondo um compromisso com a qualificação da Atenção Básica, através do trabalho da Equipe de Saúde Bucal (ESB) nas Unidades de Saúde da Família (USF)..$^{5}$

Entretanto, o profissional que tem composto estas equipes ainda vem sendo formado, em partes, segundo um modelo que privilegia o tratamento de doenças, ${ }^{6}$ trabalha de forma autônoma, não tem experiência de trabalho em equipe e tem pouca familiaridade com as instâncias do Sistema Único de Saúde (SUS), ${ }^{7}$ reproduzindo um trabalho antagônico às práticas preconizadas pela Estratégia Saúde da Família (ESF).

Em 1996, foi sancionada a Lei no. 9.394, estabelecendo as Diretrizes e Bases da Educação Nacional que, em síntese, propõe as Diretrizes Curriculares Nacionais (DCN) ${ }^{8}$ Especificamente para o curso de graduação em Odontologia, as DCN encontram-se em vigência desde 2002 e sinalizam para uma mudança paradigmática na formação, buscando materializar um profissional crítico, capaz de, dentre outros critérios, trabalhar em equipe e de levar em conta a realidade social. Propõem um cirurgião-dentista com perfil generalista, com sólida formação técnico-científica, humanística e ética, orientada para a promoção de saúde. $^{9}$

Mais do que a re-orientação de projetos pedagógicos, as DCN apontam para uma nova fase da relação entre o ensino e os serviços de saúde. Entretanto, esta relação é complexa e exige esforços intersetoriais para consolidá-la.

Nesse sentido, desponta-se a necessidade de desenvolver instrumentos que possam aprimorar a formação do futuro trabalhador em saúde. ${ }^{10}$ Entretanto, investir somente na graduação não é suficiente para formar o profissional que a sociedade precisa e que o sistema de saúde requer, ou seja, o Ministério da Saúde e da Educação precisam aproximar-se não somente através de políticas e projetos pontuais, mas de um diálogo que enfrente poderes instituídos em distintas esferas e instituições políticas, acadêmicas e dos serviços de saúde. É inerente a necessidade de buscar o engendramento de novas relações de responsabilidade e compromisso entre as instituições de ensino e o SUS, de modo que possibilite a co-gestão dos processos, para que realmente tenhamos mudanças significativas na formação em saúde. ${ }^{11}$

Dentro dessa arquitetura de reconstrução, foi pro- posto o Programa de Educação pelo Trabalho para a Saúde (PET-Saúde) uma das ações intersetoriais direcionadas à condução da interação ensino-serviçocomunidade, eixo básico para reorientar a educação na área da saúde. O programa surgiu em 2009, através de uma parceria entre a Secretaria de Gestão do Trabalho (SGTES), a Secretaria de Atenção à Saúde (SAS), o Ministério da Saúde, a Secretaria de Educação Superior (Sesu) e o Ministério da Educação. ${ }^{12}$ Caracteriza-se como uma das ações do Programa Nacional de Reorientação da Formação Profissional em Saúde (Pró-Saúde), criado em 2005, tendo como perspectiva a aproximação da formação de graduação no país às necessidades da Atenção Básica, que se traduzem no Brasil, essencialmente pela ESF. ${ }^{13}$

O PET-Saúde traz como objetivo geral fomentar a formação de grupos de aprendizagem tutorial no âmbito da ESF, denotando um instrumento para a qualificação em serviço dos profissionais da saúde, bem como para a iniciação ao trabalho e vivências dirigidos aos estudantes dos cursos de graduação na área da saúde, de acordo com as necessidades do SUS, com a perspectiva da inserção das necessidades dos serviços como fonte de produção de conhecimentos e pesquisa nas instituições de ensino. ${ }^{14}$

Esse manuscrito pretende relatar a experiência de inovação pedagógica, ocorrida com a implementação do PET-Saúde, ao longo dos seus dois anos de existência na Universidade Estadual de Feira de Santana (UEFS), Bahia. O curso de graduação em Odontologia é salientado e busca-se oferecer subsídios para que o programa seja executado e aperfeiçoado em outras localidades do país.

\section{ANSEIOS DE MUDANÇA NA FORMAÇÃO EM ODONTOLOGIA: O SUS COMO CENÁRIO DE APRENDIZAGEM}

O Curso de Graduação em Odontologia da UEFS, foi concebido em 1986 e, ao longo de sua evolução passou a almejar um profissional para atuar efetivamente na sociedade, com flexibilidade intelectual e capacidade analítica crítica, apto a interpretar a realidade social, política e cultural da região e capaz de transformá-la. No início do ano corrente, a grade curricular foi substituída por uma estrutura de currículo adequada para atender os princípios das DCN para o Curso de Odontologia.

A área de Odontologia Preventiva e Social, presente na antiga grade curricular que se encontra em processo de substituição, organiza-se de forma a articular a teoria à prática, viabilizando experiências con- 
cretas de atividades pertinentes à formação do Cirurgião-Dentista. Em 2007, a disciplina iniciou uma articulação com a ESF, tomando as USF como cenário para aprendizagem.

Não obstante, apesar de retratar um avanço significativo na condução de integração do processo de ensino-aprendizagem da graduação à rede de serviços públicos de saúde, as práticas pedagógicas desenvolvidas pelas disciplinas de Odontologia Preventiva e Social se chocam com obstáculos que diminuem a sustentabilidade, limitando a inovação pedagógica desejada. Um caminho para transformação seria o estabelecimento de vínculos mais explícitos entre o aprendizado no trabalho, na comunidade e o aprendizado nas salas de aula ${ }^{15}$.

Nesse intento, atendendo ao convite dos Ministérios da Saúde e da Educação para a apresentação de propostas com vistas à seleção dos projetos que participariam do PET-Saúde, a UEFS e a Secretaria Municipal de Saúde de Feira de Santana lograram êxito, obtendo a aprovação do PET-Saúde UEFS. Foram contemplados os cursos de graduação em Enfermagem, Medicina, Odontologia, Educação Física e Ciências Farmacêuticas, com o estabelecimento de grupos de aprendizagem tutorial vinculados à ESF. Os atores envolvidos pressentiam o PET-Saúde como uma potência para qualificar a formação profissional, reorientar práticas e provocar mudanças de concepções e atitudes com o propósito de qualificar a Atenção Básica no nível local.

\section{O TRILHAR DO PET-SAÚdE UEFS: DESCOBERTAS, TROPEÇOS E CONQUISTAS}

O PET-Saúde UEFS vem sendo implementado desde abril de 2009, quando foram iniciadas as atividades com os grupos de aprendizagem tutorial na Rede de Saúde da Família de Feira de Santana. Atualmente, o programa se encontra devidamente institucionalizado pelo Conselho Superior de Ensino, Pesquisa e Extensão da UEFS. Além disso, a UEFS é uma das instituições de ensino superior do Estado que integra a Rede Pró-Saúde Bahia, articulada pela Secretaria da Saúde do Estado da Bahia (SESAB), através da Superintendência de Recursos Humanos e da Escola Estadual de Saúde Pública (EESP) por meio da Coordenação de Integração da Educação e Trabalho na Saúde (CIET). Dessa forma, o PET-Saúde UEFS tem mantido estreita articulação com a Comissão Gestora Local do Pró-Saúde II UEFS cujos membros integram também o referido Programa.
No primeiro ano, foram formados 07 grupos de aprendizagem tutorial com um total de 07 tutores, 42 preceptores, 84 alunos bolsistas e 37 monitores. Dentre eles, 34 eram Cirurgiões-Dentistas ou graduandos em Odontologia. No ano seguinte, com a denominação de PET-Saúde da Família, o número de grupos foi elevado, passando para 10 e somando 219 atores envolvidos.

No intuito de atingir os objetivos propostos no projeto PET-Saúde UEFS, representantes das diferentes instâncias envolvidas participaram do "I Seminário Nacional do Programa Nacional de Reorientação da Formação Profissional em Saúde - Pró-Saúde II", realizado em Brasília no início de 2009. Em seguida foi realizada a "I Oficina de Mobilização para a implementação do PET-Saúde na UEFS", novamente contando com a colaboração dos diversos atores.

De forma análoga, para operacionalização do programa foi implantado o Núcleo de Excelência Clínica Aplicada à Atenção Básica (NECAAB), instalado no âmbito do Departamento de Saúde da UEFS, tendo como responsabilidades principais:

- produzir projetos de mudanças curriculares que promovam a inserção dos alunos na rede da Atenção Básica, em estreita articulação com a Comissão Gestora Local do Pró-Saúde UEFS;

- desenvolver ações para a capacitação dos preceptores dos serviços vinculados à ESF;

- incentivar e produzir pesquisa voltada para a qualificação da Atenção Básica; e

- coordenar a revisão de diretrizes clínicas da Atenção Básica, em consonância com as necessidades do SUS.

Visando favorecer o processo de inovação metodológica e incorporar novas práticas pedagógicas nos cursos articulados ao PET-Saúde, se aposta na incorporação de ações como a execução do portfólio reflexivo, instrumento construído com base em seis princípios:

- a construção pelo próprio aluno, possibilitando que ele faça escolhas e tome decisões;

- a reflexão sobre suas produções;

- a criatividade, porque o aluno escolhe a maneira de organizar o portfólio e busca formas diferentes de aprender;

- a auto-avaliação pelo aluno, porque ele está permanentemente avaliando o seu progresso;

- a parceria tutor-preceptor-aluno e entre alunos, eliminando-se ações e atitudes verticalizadas e centralizadoras; e por fim,

- a autonomia do aluno perante o trabalho. 
Outrossim, no mesmo intento, criou-se recursos de conectividade, como as listas de discussão virtual e o e-mail de acesso para todos os envolvidos, além da organização de eventos para o debate sobre a educação superior na área da saúde.

As atividades propostas e desenvolvidas pelo PETSaúde UEFS, ao longo do seu biênio de existência, priorizaram os reais problemas de saúde (situação de saúde e organização do serviço) identificados no contexto das USF, para gerar contribuições na reorientação das práticas de saúde e de gestão.

Dentre elas, estão as situadas no campo da Promoção de Saúde, entendendo-as como uma estratégia de articulação transversal que visa promover qualidade de vida e reduzir vulnerabilidades e riscos à saúde relacionados aos seus determinantes e condicionantes. Realizou-se, nesse nível, abordagens direcionadas à saúde bucal, à atividade físicas, além da discussão de temas diversos na sala de espera, de feiras de saúde, de ações de impacto, de encontros de saúde e de oficinas educativas. Ações referentes ao cuidado em saúde, como visitas domiciliares e acompanhamento da rotina das unidades, também fizeram parte do elenco de atividades desenvolvidas. As reflexões críticas sobre o trabalho em ato e as necessidades apresentadas nos processos de trabalho favoreceram a educação permanente dos profissionais e dos graduandos. Foram trabalhadas ações de vigilância em saúde, atividades gerenciais e atividades de planejamento em saúde. Além disso, pretendendo fortalecer o controle social no SUS, os grupos efetuaram um trabalho para a mobilização da comunidade. As ações executadas estão sintetizadas no Quadro I.

A despeito do curto período de existência, é pos-

Quadro I - Síntese das atividades desenvolvidas pelos grupos PET-Saúde UEFS no período de 2009 a 2010 [continua na próxima página].

\begin{tabular}{|c|c|}
\hline Tipo de abordagem & Principais atividades desenvolvidas \\
\hline Saúde bucal & $\begin{array}{l}\text { - "Dia da Escovação" com crianças das áreas de abrangência de várias USF } \\
\text { - "Dia do Sorriso" com crianças matriculadas em uma escola municipal } \\
\text { - Distribuição de kits odontológicos em diversos espaços sociais } \\
\text { - Palestras sobre doença cárie, métodos de prevenção, alimentação saudável e desenvolvimento do } \\
\text { sistema estomatognático, envolvendo escolares e professores } \\
\text { - Escovação supervisionada em escolas e creches } \\
\text { - Orientação sobre higiene bucal de bebês e crianças } \\
\text { - "Tarde animada das crianças" com atividades lúdicas } \\
\text { - Aplicação de inquérito clínico odontológico no grupo da terceira idade }\end{array}$ \\
\hline $\begin{array}{l}\text { Atividade física e } \\
\text { prática corporal }\end{array}$ & $\begin{array}{l}\text { - Atividade física e prática corporal com grupos de hipertensos, diabéticos e idosos } \\
\text { - Práticas recreativas e alongamentos com idosos } \\
\text { - Orientação sobre atividade física às gestantes } \\
\text { - "Caminhada da Saúde" } \\
\text { - Dança e ginástica } \\
\text { - Criação da horta comunitária } \\
\text { - "Forró dos idosos" }\end{array}$ \\
\hline Sala de espera & $\begin{array}{l}\text { - Atividades educativas envolvendo, além das abordagens já mencionadas, doenças como dengue, } \\
\text { tuberculose, meningite, gripe influenza A H1N1, DST/AIDS, doenças respiratórias, gravidez e parto, } \\
\text { saúde da mulher, uso inadequado de medicamentos, SUS, ESF e controle social no SUS }\end{array}$ \\
\hline Feiras de saúde & $\begin{array}{l}\text { - } 1^{a} \text { Feira de Saúde do HIPERDIA (programa direcionado aos hipertensos) } \\
\text { - Feira de Saúde do Feira X-V } \\
\text { - Feira de Saúde do George Américo } \\
\text { - Feira de Saúde do Campo Limpo I, II e IV }\end{array}$ \\
\hline $\begin{array}{l}\text { Encontros } \\
\text { de saúde }\end{array}$ & $\begin{array}{l}\text { - II Encontro de Saúde sobre Depressão } \\
\text { - I HIPERDIA da USF Feira X II } \\
\text { - Encontros em Fábricas dos bairros para discussão sobre EPI } \\
\text { - Encontro com a "Associação do Amendoim" } \\
\text { - Encontro com adolescentes - mostra de vídeo sobre gravidez na adolescência e planejamento familiar } \\
\text { - Encontro com Idosos } \\
\text { - "Roda de Terapia Comunitária" com grupos de hipertensos, diabéticos e adolescentes } \\
\text { - Encontro sobre vacinação }\end{array}$ \\
\hline
\end{tabular}


O Programa de Educação pelo Trabalho para Saúde (PET-Saúde) como indutor de inovações pedagógicas: a experiência do curso de Odontologia da Universidade Estadual de Feira de Santana, Bahia • Fonsêca GS, Rodrigues AAAO

Quadro I [Continuação].

\begin{tabular}{l|l} 
Tipo de abordagem & \multicolumn{1}{c}{ Principais atividades desenvolvidas } \\
& - Apresentação do PET-Saúde \\
& - Apresentação do Núcleo de Apoio ao Saúde da Família (NASF) \\
& - Conselho Local de Saúde \\
& - Pré-Conferência Municipal de Saúde \\
& - Um SUS pra valer e pra todos nós \\
& - Uso de contraceptivos orais e gravidez na adolescência \\
& - Levantamento de problemas com a comunidade e Agentes Comunitários de Saúde (ACS) \\
& - Atenção à Saúde do bebê \\
& - Asma brônquica \\
- Câncer de próstata & - Prevenção do câncer de mama e do colo do útero \\
- Uso de plantas medicinais & - 20 anos do SUS \\
- Cinema com pipoca para adolescentes & - Lixo: uma responsabilidade de todos
\end{tabular}

- Álbum seriado sobre cuidados com gestantes

- Cartilha do hipertenso

- Folder sobre cuidados com os pés (prevenção "do pé diabético")

- Folder sobre atividade física para gestante

- Folder sobre saúde bucal

- Folder sobre direitos e deveres dos usuários das USF

Elaboração de material educativo

- Folder sobre o SUS

- Folder sobre VDRL

- Folder sobre Métodos Contraceptivos

- Folder sobre Meningite

- Cartaz sobre saúde bucal da gestante e do bebê

- Cartaz sobre tuberculose, hanseníase e gripe A H1N1

- Cartilha de cuidados com recém nascidos

- Cartilha sobre ESF e rotina da unidade

- Visitas e consultas domiciliares

- Acompanhamento dos ACS nas visitas domiciliares

- Participação em consultas clínicas na USF

- Acompanhamento na coleta de material para exame preventivo

- Aferição de pressão arterial

Cuidado em saúde

- Dispensa de medicamentos

- Realização de curativos

- Administração de vacinas

- Pós-consulta com os pacientes do HIPERDIA

- Pré-consultas de enfermagem

- Teste do pesinho

- Remoção de sutura

- Discussão com preceptores e bolsistas

- Territorialização das áreas das USF

Planejamento em saúde

- Diagnóstico das necessidades da comunidade

- Levantamento de dados das Fichas A

- Análise dados do SIAB

- Confecção de "Parede de situação"

- Avaliação da satisfação do usuário

Atividades gerenciais

- Aplicação do instrumento de supervisão dos ACS

- Elaboração das programações mensais com as equipes das USF

(continua) - Planejamento de ações com ACS

- Reorganização das farmácias das USF 
O Programa de Educação pelo Trabalho para Saúde (PET-Saúde) como indutor de inovações pedagógicas: a experiência do curso de Odontologia da Universidade Estadual de Feira de Santana, Bahia • Fonsêca GS, Rodrigues AAAO

Quadro I [Continuação].

\begin{tabular}{|c|c|}
\hline Tipo de abordagem & Principais atividades desenvolvidas \\
\hline $\begin{array}{l}\text { Atividades } \\
\text { gerenciais } \\
\text { (continuação) }\end{array}$ & $\begin{array}{l}\text { - Preenchimento do mapa de solicitação de medicamentos } \\
\text { - Organização dos arquivos e documentos das USF } \\
\text { - Preenchimentos das fichas do SIAB e das fichas de referência e contra-referência } \\
\text { - Supervisão de ACS e técnicos de enfermagem } \\
\text { - Consolidação dos dados das USF } \\
\text { - Formalização de protocolos de rotinas das USF } \\
\text { - Reunião para avaliação trimestral do PET-Saúde, envolvendo todos os atores } \\
\text { - Visitas técnicas ao Hospital Geral Clériston Andrade, à Policlínicas e ao Centro Municipal de Referência } \\
\text { em Hipertensão e Diabetes }\end{array}$ \\
\hline Vigilância em saúde & $\begin{array}{l}\text { - Investigação de casos notificados de dengue } \\
\text { - Investigação de óbito neonatal } \\
\text { - Busca dos idosos faltosos da campanha de vacinação contra gripe A H1N1 }\end{array}$ \\
\hline $\begin{array}{l}\text { Estudo e discussão } \\
\text { em grupo }\end{array}$ & $\begin{array}{l}\text { - SUS: Histórico e evolução das Políticas de Saúde } \\
\text { - Política Nacional de Atenção Básica } \\
\text { - ESF; Organização e funcionamento das USF } \\
\text { - O processo de territorialização } \\
\text { - Vigilância em Saúde } \\
\text { - Planejamento e Programação em Saúde } \\
\text { - Trabalho em equipe multiprofissional } \\
\text { - Processo de Trabalho em Saúde } \\
\text { - Saúde do Trabalhador } \\
\text { - Visitas domiciliares } \\
\text { - Tecnologias leves em educação e em saúde } \\
\text { - Uso do portfólio } \\
\text { - Busca bibliográfica através das principais de bases de dados } \\
\text { - Elaboração de projetos de pesquisa } \\
\text { - Portaria } 154 / 2008 \text { - NASF }\end{array}$ \\
\hline
\end{tabular}

sível descrever alguns avanços oriundos da atuação do PET-Saúde. Dentre eles, menciona-se a notória produção científica que vem sendo divulgada a nível local, regional, nacional e internacional, representando um incremento significativo nas pesquisas relacionadas à Saúde Coletiva. Diversos projetos envolvendo parcerias do PET-Saúde com componentes curriculares, núcleos de pesquisa e projetos de extensão foram ou estão sendo desenvolvidos.

Uma avaliação processual revela indicativos de impactos sobre os problemas de saúde identificados. Eles serão expostos minuciosamente em uma avaliação de resultados que será realizada futuramente pelos integrantes do PET-Saúde.

No decorrer do processo de implantação, os envolvidos se depararam com limites para o estabelecimento pleno das diretrizes de funcionamento do programa devido ao seu pioneirismo. Majoritariamente, esses limites estão ligados à preceptoria que demonstra dificuldade em compatibilizar os horários entre os estudantes; falta de experiência com a condução de projetos de pesquisa; perfil inadequado para o trabalho na ESF e dificuldade de orientação quando havia o envolvimento de áreas do conhecimento distintas entre preceptor e graduando. Além disso, um complicador significativo na condução das atividades correspondeu à infra-estrutura deficiente das USF e a resistência por parte de alguns integrantes das equipes de saúde com relação ao trabalho do PET-Saúde.

\section{CONClusões}

Realizando uma retrospectiva dos dois anos de existência do PET-Saúde UEFS, observa-se que as atividades desenvolvidas trazem expressiva relevância para a formação e a qualificação profissional dos participantes nos aspectos teóricos e práticos. Percebe-se a aquisição de habilidades importantes direcionadas ao efetivo labor na ESF e o aumento da experiência com a elaboração de projetos de pesquisa.

É evidente que o estabelecimento do PET-Saúde como uma das alternativas de consolidação do SUS requer a participação ativa de atores sociais comprometidos ética e politicamente com a (re)construção 
coletiva de saberes e práticas, e também com a superação de problemas conjunturais que envolvem a institucionalização e a consolidação de um programa novo e inovador que está sendo construído por sujeitos sociais que optaram por uma proposta dinâmica de formação através do trabalho em saúde, usando como ferramenta a educação permanente, onde se crê que reside toda capacidade de transformação de antigos paradigmas desta área em posturas e atitudes mais coerentes com as propostas do SUS, alimentando o desejo de construir um sistema público de saúde mais efetivo, rompendo inclusive com padrões históricos e tradicionais do "processo ensino-aprendizagem".

Nesse sentido, é perceptível que as atividades desenvolvidas pelo PET-Saúde UEFS priorizam formar profissionais mais decisivos e desenvoltos, com conhecimentos e atividades que os tornem competentes para atuar no SUS, com amadurecimento profissional e elevada formação científica, tecnológica e humanística.

É indiscutível que o PET-Saúde constitui uma excelente proposta de integração entre o trabalho em saúde e a educação, propiciando a articulação entre o ensino, a pesquisa e a extensão na área de saúde coletiva, com foco na Atenção Básica/ESF.

Os significativos resultados obtidos e os movimentos articulados pelo programa nos seus primeiros anos de atuação, demonstram que o PET-Saúde UEFS/ SMS-FS encontra-se devidamente implementado com suporte operacional e apoio político-institucional (UEFS/SMS/CMS/SESAB/MS), fatores que o qualificam para a sua tão almejada expansão.

\section{ABSTRACT}

The Education Program through Health Work as an inducer of pedagogical innovations: the Dentistry Course experience at the State University of Feira de Santana, Bahia

The development of dental practices and the new concepts regarding the effectiveness of oral healthcare require professionals to be critical, able to work in teams and able to assimilate the social reality. What is proposed is to train dentists with a generalist profile, with solid technical and scientific skills, who are humanistic and ethical, and also focused on promoting health. With this in mind, changes are being implemented in higher education, seeking integration with health services and the community, in order to promote the teaching-learning process. In this context, the Education Program through Health Work is con- sidered a strong inducer of pedagogical innovation. This paper describes the experience of the State University of Feira de Santana, Bahia, highlighting the changes made in the dentistry course, in order to provide input for supporting the implementation and improvement of the program in other parts of the country. It could be observed that significant results were achieved by incorporating the Education Program through Health Work, showing its transformational potential, which promotes its consolidation and expansion.

\section{DESCRIPTORS}

Higher education. Health services. Dentistry.

\section{REFERÊNCIAS}

1. Pinto VG. A Odontologia brasileira às vésperas do ano 2000: diagnóstico e caminhos a seguir. São Paulo: Santos; 1993. 189p.

2. Haddad AE, Morita MC. O Ensino de Odontologia e as Políticas de Saúde e de Educação. In: Perri de Carvalho AC, Kriger L, editores. Educação Odontológica. São Paulo: Artes Médicas; 2006. p.105-17.

3. Brasil. Ministério da Saúde. Secretaria de Atenção à Saúde. Departamento de Atenção Básica. Projeto SB 2003: Condições de Saúde Bucal da população brasileira 2002-2003: resultados principais. Brasília 2004.

4. Narvai PC, Frazão P. Políticas de Saúde Bucal no Brasil. In: Moysés ST, Kriger L, Moysés SJ. Saúde Bucal das Famílias: Trabalhando com evidências. São Paulo: Arte Médicas; 2008, p.0120 .

5. Brasil. Conselho Nacional de Secretários de Saúde. Diretrizes da Política Nacional de Saúde Bucal. Brasília 2004.

6. Rodrigues AAAO, Fonsêca GS, Siqueira DVS, Assis MMA, Nascimento MAA. Práticas da Equipe de Saúde Bucal na Estratégia Saúde da Família e a construção (des) construção da integralidade em Feira de Santana - BA. Rev APS. 2011,13:(4):476-85.

7. Morita MC, Kriger L. Mudanças nos cursos de Odontologia e a interação com o SUS. Revista da ABENO. 2004, 4:(1):17-21.

8. Brasil. Ministério da Saúde. Lei n. 9.394, 20 dezembro de 1996 Institui a Lei de Diretrizes e Bases da Educação Nacional. Brasília, 1996.

9. Brasil. Ministério da Educação. Conselho Nacional da Educação. Câmara da Educação Superior. Resolução CNE-CES n. 3. Instituiu as Diretrizes Curriculares Nacionais do Curso de Graduação em Odontologia. Brasília 2002.

10. Tavares DMS, Simões ALA, Poggetto MTD, Silva SR. Interface ensino, pesquisa, extensão nos cursos de graduação da saúde na Universidade Federal do Triângulo Mineiro. Rev Lat Am Enfermagem. 2007,15(6):1080-5.

11. Garcia RA, Carvalho SR. Navegando no entre das Instituições de Ensino e Serviços de Saúde: Uma carta náutica dos (des) 
O Programa de Educação pelo Trabalho para Saúde (PET-Saúde) como indutor de inovações pedagógicas: a experiência do curso de Odontologia da Universidade Estadual de Feira de Santana, Bahia • Fonsêca GS, Rodrigues AAAO

encontros. In: Carvalho SR, Ferigato S, Barros ME, editores. Conexões: Saúde Coletiva e Políticas de Subjetividade. São Paulo:Hucitec; 2009. p. 220-39.

12. Brasil. Ministério da Saúde. Ministério da Educação. Portaria Conjunta n. 3. Homologa o resultado do processo de seleção dos Projetos que se candidataram ao Programa de Educação pelo Trabalho para a Saúde: PET-Saúde. Brasília 2009.

13. Brasil. Ministério da Saúde. Ministério da Educação. Secretaria de Gestão do Trabalho e da Educação na Saúde. Secretaria de Educação Superior. Instituto Nacional de Estudos e Pesquisas Educacionais Anísio Teixeira. Programa Nacional de Reorienta- ção da Formação Profissional em Saúde: Pró-Saúde. Brasília 2005.

14. Brasil. Ministério da Saúde. Ministério da Educação.Portaria Interministerial n. 1.802, 26 de agosto 2008. Institui o Programa de Educação pelo Trabalho para a Saúde: PET-Saúde. Brasília 2008.

15. Young MFD. O currículo do futuro: da "nova sociologia da educação" a uma teoria crítica do aprendizado. Campinas: Papirus; 2000. 288 p.

Recebido em 07/07/2011

Aceito em 25/07/2011 